\title{
Molecular dynamic simulation of carbon nanotube reinforced nanocomposites: the effect of interface interaction on mechanical properties
}

\begin{abstract}
The mechanical properties of single wall carbon nanotube reinforced polymer (CP) composite have been investigated using COGNAC in molecular dynamic (MD) simulations. Nanocomposites are constructed by embedding single wall CNT $(5,5)$ into acrylate based polymer under the periodic boundary condition with different interface interaction. Nanocomposites systems underwent NPT (constant number of atoms, pressure and temperature) and NVT (constant number of atoms, volume and temperature) ensemble with applied uniform strain during the MD simulations. The effect of the quality of filler- matrix interface on the mechanical properties was evaluated by altering the van der Waals interaction energy potential between the polymer and the filers. It is demonstrated that in spite of the presence of different interface interaction, Young's modulus of the pure polymer is enhanced by incorporating CNT and leads to mechanical properties exceeding the unfilled polymer. The tensile strength and the ultimate stress also follow similar tendencies. The results demonstrate that, there may be a reasonable relationship between the interfacial bonding and the load transfer between the matrix and fillers which has great impact on the mechanical properties of the nanocomposites. It was shown that by increasing the interface interaction, the opposite effects are observed in Young's modulus, tensile strength and the ultimate stress point. The observation confirms that the incompressibility of the interface region under the optimum interface interaction leads to the optimum mechanical properties.
\end{abstract}

Keywords: MD simulation, nanocomposites, carbon nanotubes, interface interaction, mechanical property

\author{
Volume 2 Issue I - 2018
}

\author{
Ommeaymen Sheikhnejad,' Takahiko \\ Nakamoto, ${ }^{2}$ Anna Kalteis, ' Ali Rajabtabar, ${ }^{3}$ \\ Zoltan Major' \\ 'Institute of Polymer Product Engineering, Johannes Kepler \\ University Linz, Austria \\ ${ }^{2}$ Institute of Strength and Mechanism in Design, Kanazawa \\ University, Ishikawa, Japan \\ ${ }^{3}$ Institute of Biophysics, Johannes Kepler University Linz, Austria
}

Correspondence: Ommeaymen Sheikhnejad, Institute of Polymer Product Engineering, Johannes Kepler University Linz, Austria, Email ommeaymen.sheikhnejad-bishe@jku.at

Received: January 04, 2018 | Published: January 18, 2018
Abbreviations: MD, molecular dynamic; CNT, carbon nano tubes; LDI, lysine-based diisocyanate; RVE, representative volume elements

\section{Introduction}

Polymer nanocomposites are known to exhibit enhanced mechanical properties compared to neat polymeric system. ${ }^{1}$ Fillers can be used as reinforcement in polymer composites for the enhancement of mechanical properties. ${ }^{2}$ Reinforced" polymers consist of a polymeric matrix and relatively stiff filler that exhibit a drastic change in modulus or stress at given strain in compare with the pure polymer. ${ }^{3}$

Researchers have been incorporating different kinds of nanofillers in order to achieve better properties based on the application. In context, carbon nanotubes (CNT) attracted a growing interest of research community and industry in recent years. They have shown promising due to their remarkable mechanical, electrical and thermal properties as well as plus light weight, which promise wide range of their potential applications in nano devices. ${ }^{4,5}$ In order to fulfill the promises, some critical issues must be fully understood. ${ }^{6}$ One of the most significant challenges towards improving the properties of the nanocomposites is to control the interaction at the interface of the constituent materials, which determines the efficiency of load transfer from the polymer matrix to the fillers. ${ }^{7}$ The mechanical properties are largely governed by the interfacial interaction and the lack of optimum interfacial interaction between the filler and the polymeric matrix leads to weak interfacial adhesion and results in failure. ${ }^{3}$ However, the interfacial interaction is also the weakest part in the composites, which can be attributed to the different mechanical properties between matrices and fillers because of the formation of an interphase region in the matrix close to the surface of the fibers. ${ }^{8}$ For this purpose, MD simulations have been applied, which provide a powerful tool for characterizing the relationship between structure and properties and also enable us to focus on the behavior of the interface region between filler and matrix. ${ }^{9}$

Lee et al. ${ }^{10}$ studied the effects of lysine-based diisocyanate (LDI) on the properties of biocomposite. They suggested that both PLA/ $\mathrm{BF}$ and $\mathrm{PBS} / \mathrm{BF}$ composites yield better tensile properties under the improved interfacial adhesion. Mccarthy et al. ${ }^{11}$ examined the interaction between carbon nanotubes and a conjugated polymer through microscopic and spectroscopic techniques. They concluded that a good wetting between the conjugated polymer and nanotubes, the nucleation of crystalline growth by defects in the nanotube, and a structured wrapping of the conjugated polymer onto the nanotube lattice.

In this work, we seek to develop molecular dynamic simulation method to investigate the role of CNT on the mechanical properties of the polymer nanocomposites. The objective is to develop a computational molecular dynamic model involving single wall CNT in polymer in order to characterize the elastic behavior of the nanocomposites and to explore factors controlling the strength in nanofillers reinforced nanocomposites using JOCTA software. Molecular model of the nanocomposites were constructed by 
reinforcing acrylate based polymer with CNT as nanofillers. In terms of comparison between simulated nanocomposites, the size of the representative volume elements (RVE), matrix characteristics and applied loading were kept constant. In particular, we focused on the nanoparticles/polymer interface effects by altering the van der Waals interaction between the polymer and nanofiller. In order to take into account the interface effect, we employed so-called "strong and weak interaction models", in which the interaction between the matrix and nanofiller is varied. Molecular model for the pure matrix was also simulated to have better understandings of the effect of nanofillers on the mechanical properties of the nanocomposites.

\section{Molecular dynamic modelling and simulation}

The molecular dynamic simulations were carried out using COGNAC in J-OCTA (JSOL Corporation) package. Periodic boundary conditions were applied in all nanocomposites to replicate the cell in all three dimensions and improve the rigor and realism of simulated models. ${ }^{7}$ All simulations were carried out in two main steps which called as relaxation and loading step and they provide the equilibrium state and the deformed structure of the molecular model respectively.

First, the representative volume element (RVE) of the pure polymer was modeled based on isobornyl acrylate monomers with amorphous molecular structure and the RVE geometry of the molecular model selected was a cubic box. ${ }^{12}$ Neat polymer model was built with 292 units of monomer in a periodic box (Figure 1) and using DREIDING force field parameters to establish the bond, angle and dihedral parameters. $^{13}$ (a)

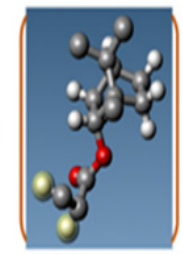
$n=292$

(b)
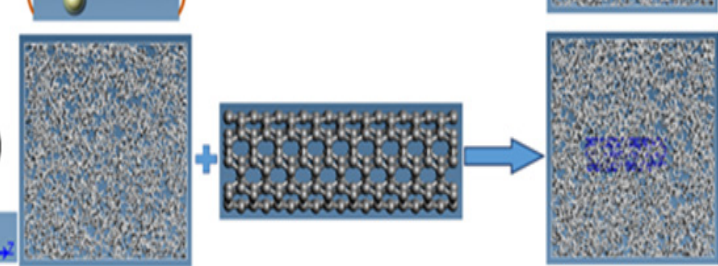

(c)

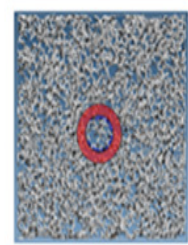

Figure I MD snapshots of: (A) Isobornyle acrylate structure as a monomer for constructing neat polymer, (B) CNT-polymer (CP) nanocomposite, (C) the red circle shows the effective interfacial zone between CNT-polymer.

The relaxation state in MD simulation implies obtaining fully relaxed (stress-free) state for the corresponding atomistic model..$^{12}$ In this research paper, the equilibrium step for all the simulated models was achieved by running simulation in NPT condition (constant number of atoms, pressure and temperature). For this purpose the model was put into an NPT ensemble simulation with a pressure of $1 \mathrm{~atm}$ and temperature of $300 \mathrm{~K} \cdot{ }^{14}$ As a result, the simulated system was to slowly compress the structure to generate initial amorphous matrix with the correct density and low residual stresses..$^{15}$ After the equilibrium step, the pure matrix structure was built by replication in a periodic box. Mechanical performance of polymer nanocomposites is dependent upon the interfacial interaction between the nanofiller and polymer matrix. ${ }^{3}$ So, increasing attention is being focused on the CNT surface, namely the interface between the CNT and surrounding polymer matrix. ${ }^{14}$ Based on the description, nanocomposites were constructed using three different models with different interface interaction. First, the molecular models of polymer nanocomposites were designed by embedding CNT in the pure acrylate based matrix.

For each model, the simulation RVE with a size of $49.69 \mathrm{~nm} \times 46.35 \times 45.91$ is constructed and the periodic boundary condition is applied in all three dimensions The single wall CNT model was built with 190 atoms with a chiral index of $(5,5)$ and has a length of $16.6 \mathrm{~nm}$ and diameter of $5.9 \mathrm{~nm}$. The atomistic models of the simulated nanocomposites are shown in Figure 1. The second step is to build an equilibrated structure of filler-matrix under periodic boundary condition in order to obtain free stress state.

Afterwards, the simulated RVEs were subjected uniaxial deformation using NVT (constant number of atoms, volume and temperature) simulation and the constant strain was applied along $\mathrm{z}$ direction. The stress-strain curves of the simulated nanocomposites under longitudinal loading direction were also calculated. The elastic modulus was computed from the linear regime of the stress-strain curve. Figure 2 shows the comparison of the stress- strain curves for neat polymer and simulated nanocomposites. Then, in order to take into account the interface effect, the interface energy of the $\mathrm{CP}$ nanocomposite increased (a) 10 times (CP-10) and (b) 100 times (CP-100) and the Young's modulus and ultimate stress point for both models have been investigated.

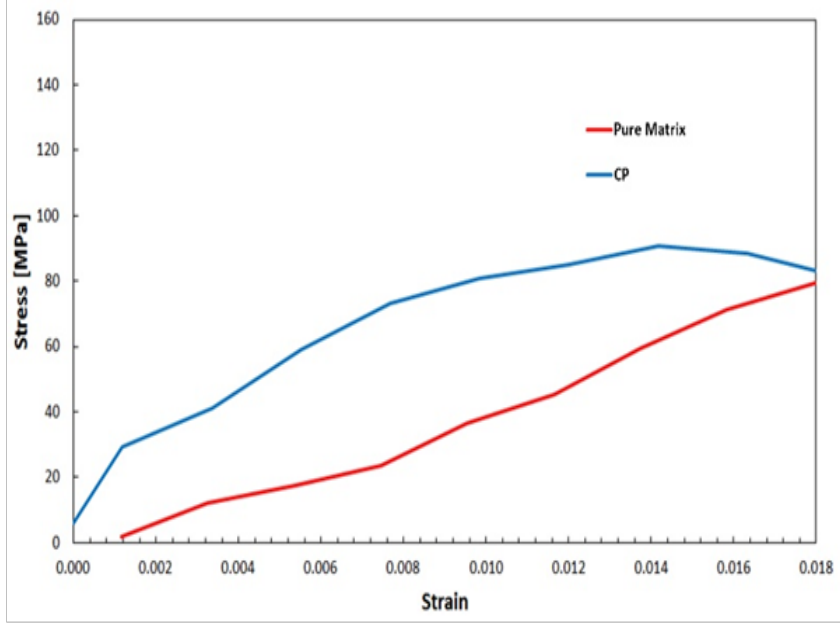

Figure 2 Stress-strain curves of pure matrix and CP nanocomposite.

\section{Results and discussion}

\section{Effect of CNT on stress- strain relations}

In this section, the effect of CNT as a filler on the mechanical properties of nanocomposites is studied and the overall Young's modulus of each model was obtained from the plot of stress-strain curve. The stress- strain curve of the CP nanocomposite generated from MD simulation under longitudinal loading condition is presented in Figure 2. In order to study the impact of CNT on the nanocomposite 
properties, the stress- strain curve of the pure matrix was also exhibited. The endpoints of the curves indicate that how far the simulation was conducted and should not consider it as yield points. ${ }^{16}$ Both curves can be regarded as straight lines which indicate that elastic deformations occurred for all structures within the strain range of $0-0.018 .{ }^{9}$ Comparing the CNT reinforced nanocomposite with pure polymer, one can see that the tensile strength increases by adding the filler in to the pure matrix. The Young's modulus of nanocomposites also increases from $4.37 \mathrm{GPa}$ (Pure matrix) to $7.67 \mathrm{GPa}(\mathrm{CP})$, with the increase of $57 \%$ (Table 1). It can be seen that by addition of CNT as a filler, the ultimate stress point is drastically increased from which implied that $\mathrm{CP}$ can handle higher loading than the pure matrix.

This study depicts a very useful picture of the influence of CNT on the mechanical properties of the nanocomposites. It proposed that CNT reinforcement has a very strong impact on the mechanical properties of nanocomposite and the stiffness enhancement effect by addition of CNT is significant. ${ }^{17}$

\section{Effect of interface interaction on mechanical proper- ties}

In order to take a closer look at the effect of interface interaction on the mechanical properties of the nanocomposites, we have modified the interface attraction between CNT and the matrix. For this purpose, the interface interaction between the $\mathrm{CNT}$ and the matrix was increased 10(CP-10) and 100(CP-100) times and the results are compared with CP. These discussions are followed by a comparison of the stress-strain curve, Young's modulus and ultimate stress point of these three model systems. ${ }^{18}$ In terms of the better understanding of the simulated nanocomposites, the MD snapshots of all three models are shown before and after deformation (Figure 3).

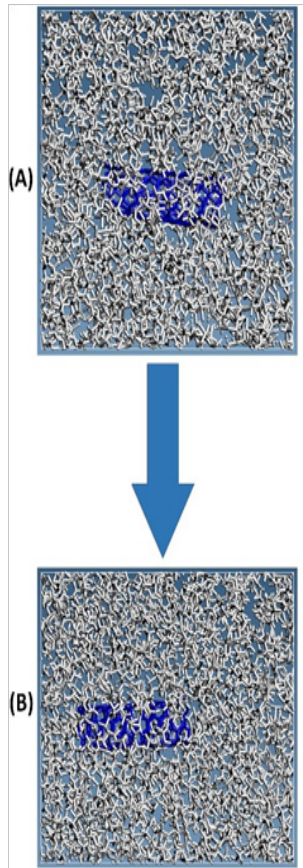

(a)
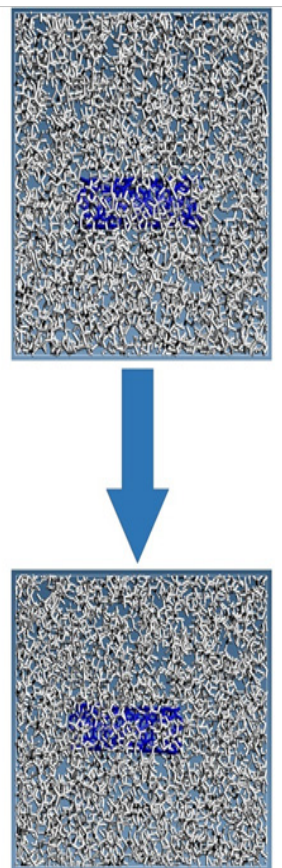

(b)
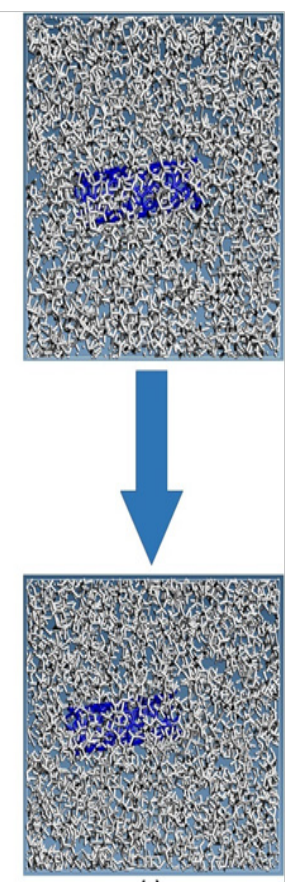

(c) interaction between filler and matrix. The elastic response of these nanocomposites is compared with the elastic property of $\mathrm{CP}$ to allow for a more representative comparison.

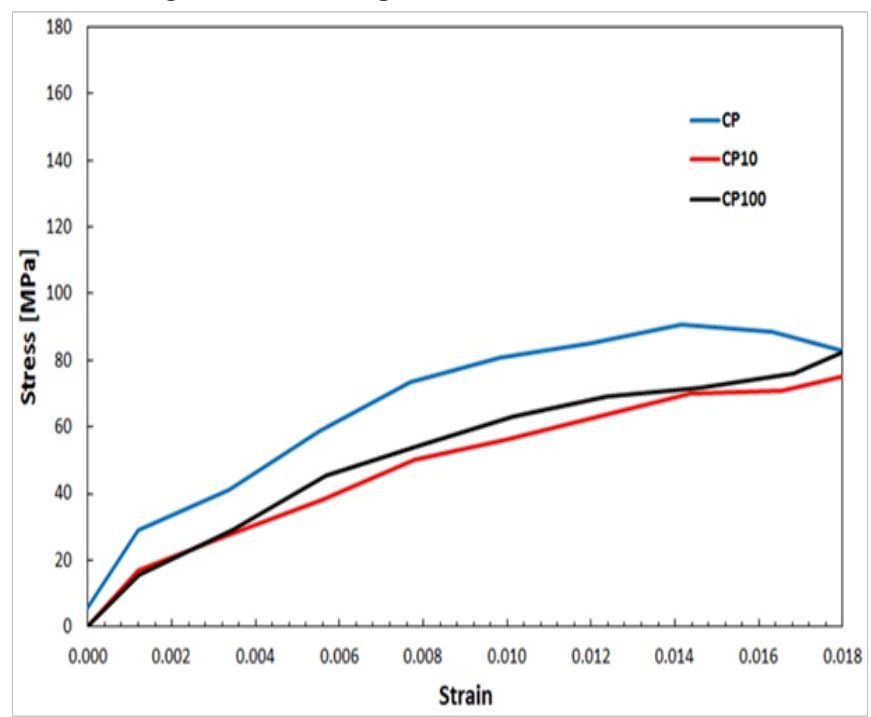

Figure 4 Stress-strain curves of CP, CP-10 and CP- 100 nanocomposite.

Based on the results, almost no enhancement is observed in the stress-strain curve of the nanocomposites with the stronger interface interaction. The value for Young's modulus and ultimate stress for CP10 and CP-100 are considerably lower than CP which are illustrated in Table 2.

Table I Evaluated modulus of elasticity and the ultimate stress point for pure matrix and $\mathrm{CP}$

\begin{tabular}{lll}
\hline Model & $\begin{array}{l}\text { Modulus of elasticity } \\
(\mathbf{G P a})\end{array}$ & $\begin{array}{l}\text { Ultimate stress } \\
(\mathbf{M P a})\end{array}$ \\
\hline Pure matrix & 4.37 & 79 \\
$\mathrm{CP}$ & 7.62 & 91 \\
\hline
\end{tabular}

Table 2 Evaluated modulus of elasticity and the ultimate stress point for $C P$, CP-I0 and CP-I00 nanocomposite

\begin{tabular}{lll}
\hline Model & $\begin{array}{l}\text { Modulus of elasticity } \\
(\mathbf{G P a})\end{array}$ & $\begin{array}{l}\text { Ultimate stress } \\
(\mathbf{M P a})\end{array}$ \\
\hline $\mathrm{CP}$ & 7.62 & 91 \\
$\mathrm{CPIO}$ & 4.86 & 71 \\
$\mathrm{CPI00}$ & 4.92 & 76 \\
\hline
\end{tabular}

These findings confirm that the interface interaction between CNT and polymer matrix affects the load transfer from polymer to the filler and has a great influence on the mechanical properties of the nanocomposites. ${ }^{19}$ The polymer at the interface can significantly contribute to the mechanical properties of the nanocomposites. ${ }^{20}$ The results support the idea that, there are several competing factors involving in the load transfer from matrix to the CNT such as matrix density and the property of the interface. CNTs are well-known because of their high effective surface area per volume, which is called as specific surface area. ${ }^{21}$ By introducing the stronger interface, more polymers are adsorbing to the interface and the density of the

Figure 4 shows the stress- strain curves from uniaxial tensile test on the simulated nanocomposites as a function of interface 
polymer in the surrounding region becomes low. On the other hands, the strong bonding with high density at the interface region caused the compressible interface region and caused an effect of pinning the matrix in the interface region. As a result, the motion of the polymer chains is limited and the reinforcement of the matrix due to the stiff filler is negated. ${ }^{22}$ It is evident in the MD snapshots that for the higher interface interaction (CP-10 and CP-100) that, there will be cavitation in the matrix after elongation but for $\mathrm{CP}$, matrix cavitation and interface failure are observed. ${ }^{1}$ As shown in Figure 4 , the only difference between these three nanocomposites is the nature of the matrix-filler interface and the higher value of Young's modulus for CP nanocomposite can be directly attributed to the higher incompressibility of the CNT- polymer interface which accelerates the load transfer from matrix to the filler.

\section{Conclusion}

In this research, the effect of CNT reinforcement on the stress-strain behavior of the nanocomposites was investigated by atomistic MD simulations. Moreover, the impact of interface interaction between CNT-polymer on mechanical properties of the nanocomposites was also evaluated.

The simulated model incorporated the molecular structures of the pure acrylate based matrix, CNT and the interfacial regions with different interfacial interactions. All the modeled nanocomposites were subjected to longitudinal loading in z-direction and the boundary condition were applied to all of them. Increases in all nanocomposites moduli with different interface interaction are almost observed in comparison with the pure matrix, which implies the reinforcement effect of the filler. The results confirm that, the interface interaction has great influence on the load transfer between matrix and the filler. The results indicate that, the stronger the interface interaction between the CNT and the polymer matrix, the lower the Young's modulus as a result of the compressibility of the interface region.

It is concluded that the presence of optimum interface interaction between the polymer and CNTs is anticipated to lead for further enhancement of stress-strain curve. While the fluctuation of density of matrix is hindered by the strong adsorption of the partial polymers as a result of strong interaction between matrix and filer. On the other hand, the weak interface interaction between matrix and filler leads to the significant decrease in the stiffness of the nanocomposite and results in low mechanical performance of the simulated nanocomposite.

We suggest that the optimum interface interaction as long as the CNT maintains its structural integrity is one of the key contributors in improving the mechanical properties of the nanocomposites.

\section{Acknowledgements}

None.

\section{Conflict of interest}

The author declares no conflict of interest.

\section{References}

1. Rahman R, Haque A. Molecular dynamic simulation of graphene reinforced nanocomposites for evaluating elastic constants. Procedia Engineering. 2013;56:789-794.
2. Dai G, Mishnaevsky L. Graphene reinforced nanocomposites: 3D simulation of damage and fracture. Computational Materials Science. 2014;95:684-692.

3. Bhattacharya M. Polymer nanocomposites-A comparison between carbon nanotubes, graphene, and clay as nanofillers. Materials. 2016;9(4):262.

4. Moradi M, Kateb M, Aghazadeh J. Mechanical properties of carbon nanotubes and graphene junction: a molecular dynamics simulation. $4^{\text {th }}$ International conference on ultrafine grained and nanostructured materials, Tehran, Iran; 2013

5. Andrews, Weisenberger M. Carbon nanotube polymer composites. Current Opinion in Solid State and Materials Science. 2004;8(1):31-37.

6. Cao G. Atomistic studies of mechanical properties of graphene. Polymers. 2014;6(9):2404-2432.

7. Gou J, Minaie B, Wang B. Computational and experimental study of interfacial bonding of single-walled nanotube reinforced composites. Computational Materials Science. 2004;31(3-4):225-236.

8. Yu K, Wang M, Wu J, et al. Modification of the interfacial interaction between carbon fiber and epoxy with carbon hybrid materials. Nanomaterials. 2016;6(5):89.

9. Izumi A, Nakao T, Shibayama M. Atomistic molecular dynamics study of cross-linked phenolic resins. Soft Matter. 2012;8(19):5283-5292.

10. Lee SH, Wang S. Biodegradable polymers/bamboo fiber biocomposite with bio-based coupling agent. Composites Part A: Applied Science and Manufacturing. 2006;37:80-91.

11. Mccarthy B, Coleman J, Czerw R, et al. A microscopic and spectroscopic study of interactions between carbon nanotubes and a conjugated polymer. The Journal of Physical Chemistry B. 2002;106(9):2210-2216.

12. Valavala P, Clancy T, Odegard G, Gates TS. Nonlinear multiscale modeling of polymer materials. International Journal of Solids and Structures. 2007;44(3-4):1161-1179.

13. Aluko O, Gotham S, Odegard G. Molecular dynamics model of graphene nanoplatelet in EPON 862/DETDA polymer. In: proceedings of the American society for composites: thirty-first technical conference; 2016.

14. Ferdous SF, Sarker MF, Adnan A. Role of nanoparticle dispersion and filler-matrix interface on the matrix dominated failure of rigid C60-PE nanocomposites: A molecular dynamics simulation study. Polymer. 2013;54(10):2565-2576.

15. Han Y, Elliott J. Molecular dynamics simulations of the elastic properties of polymer/carbon nanotube composites. Computational Materials Science. 2007;39(2):315-323.

16. Frankland S, Harik V, Odegard G, et al. The stress-strain behavior of polymer-nanotube composites from molecular dynamics simulation. Composites Science and Technology. 2003;63(11):1655-1661.

17. Mirjalili F, Chuah L, Salahi E. Mechanical and morphological properties of polypropylene/nano $\alpha-\mathrm{Al}_{2} \mathrm{O}_{3}$ composites. The Scientific World Journal Article. 2014;ID:718765 p. 12.

18. Khare KS, Khabaz F, Khare R. Effect of carbon nanotube functionalization on mechanical and thermal properties of cross-linked epoxy-carbon nanotube nanocomposites: role of strengthening the interfacial interactions. ACS Appl Mater Interfaces. 2014;6(9):6098-6110.

19. Zakaria AZ, Shelesh-Nezhad K. The effects of interphase and interface characteristics on the tensile behaviour of $\mathrm{POM} / \mathrm{CaCO}_{3}$ nanocomposites. Nanomaterials and Nanotechnology. 2014;4:17. 
20. Falkovich SG, Nazarychev VM, Larin SV, et al. Mechanical properties of a polymer at the interface structurally ordered by graphene. The Journal of Physical Chemistry C. 2016;120(12):6771-6777.

21. Liu H, Brinson LC. Reinforcing efficiency of nanoparticles: a simple comparison for polymer nanocomposites. Composites Science and Technology. 2008;68:1502-1512.
22. Zhu R, Pan E, Roy A. Molecular dynamics study of the stress-strain behavior of carbon-nanotube reinforced Epon 862 composites. Materials Science and Engineering: A. 2007;447:51-57. 